\title{
DISTRIBUTED PRODUCTION WITH SPECIFICATION-GENERATED PROCESSES
}

\author{
Tomasz Janowski \\ The United Nations University \\ International Institute for Software Technology \\ P.O.Box 3058, Macau, China \\ tj@iist.unu.edu
}

\begin{abstract}
We study design support for a virtual organization: how to organize production among the set of distributed cells to meet a given production goal. Each cell has a certain capacity to store, manufacture and deliver products, and interacts with other cells by means of information and logistics networks. The goal specifies the product and the volume required. To solve this problem we propose a protocol which given a goal and a cell attempts to generate a sequence of operations on the cell to implement this goal. Lacking the resources locally the protocol generates some auxiliary goals which it communicates to other cells, involving generating (recursively) more processes and goals etc. We formalize the problem and its solution, offer correctness arguments and discuss an email-based implementation.
\end{abstract}

\section{INTRODUCTION}

Industrial virtual organizations are no longer just a subject of academic study, they are a fact of life (Handfield and Nichols, 1999). They emerged as soon as the technological progress, in particular information and communication technology made them possible. Yet behind this application-driven progress, we have seen rather less progress in methodologies (methods, notations and tools) to guide the design of such organizations (Vernadat, 1996). Without such a methodology it is hard to realize what kind of objectives the organization is supposed to meet, does it meet those objectives, what design decisions can be made, what are the tradeoffs attached with each etc. One research direction is trying to automate as much as possible of the design effort which comes to building a virtual organization, by: formulating relevant problems, proposing algorithmic solutions, arguing (formally or informally) about their correctness, finally providing implementations.

In this paper we investigate the problem of distributed production planning to meet a certain production goal. The goal includes the type of the product and the volume required (number of items). As usual, to meet this goal the virtual organization is not built from the scratch but based on the set of existing organizations, each modeled as a single production cell. A cell has a certain capacity 
to store, manufacture and deliver products. The shopfloor (manufacture) capacity is static while the product stocks are dynamic. The cell carries out production internally, based on the existing stocks/shopfloor, and also interacts with other cells by means of information and logistics networks. We provide the formalization of this problem, its constraints and objectives, and propose an algorithmic solution.

The solution consists of a process-generator, which given a cell and a goal attempts to built a process (a sequence of operations) to be carried out by the cell to meet this goal. Lacking the resources locally the generator may also output some auxiliary goals. Then in order for the cell to meet the original goal, other cells must satisfy those auxiliary goals. The protocol communicates such goals over the information network, trying to find a match between them and the cells. This in turn involves the work of the process-generator in each individual cell, therefore producing more processes and possibly more auxiliary goals. This procedure is recursive in nature, however with each turn the goals become generally "simpler", in terms of the volumes (smaller) or products (lower in the bill-of-products hierarchy) involved, until a cell is found which implements them entirely on its own. The protocol fails to find the right match between the cells and the goals when it lacks the resources among all of the cells (globally). We formalize this solution, justify why it correctly solves the problem and propose an email-based implementation.

The rest of this paper is as follows. Section 2 introduces the notation and provides the basic concepts for production modeling. The notation is a fragment of the RAISE Specification Language (The RAISE Method Group, 1992). Section 3 formulates the problem of distributed production planning (Section 3.1), proposes a solution (Section 3.2), argues about its correctness (Section 3.3) and describes an email-based implementation (Section 3.4). Section 4 presents some conclusions.

\section{MODELING PRODUCTION, SYMBOLICALLY}

Modeling production starts with modeling products. The type Product represents all products. We have two functions on this type: size returns a number representing the storage requirements of a product and bill returns all sub-products with their quantities (to obtain a single item of the product). Both are declared as values of the corresponding functional types, the return type of size is Nat (natural numbers), the return type of bill is Product $\stackrel{m}{\longrightarrow}$ Nat (finite maps from Product to Nat).

\begin{tabular}{l|l}
$\begin{array}{l}\text { type } \\
\text { Product }\end{array}$ & $\begin{array}{l}\text { value } \\
\text { size: Product } \rightarrow \text { Nat, } \\
\text { bill: Product } \rightarrow \text { (Product } \stackrel{m}{\longrightarrow} \text { Nat) }\end{array}$
\end{tabular}

We also introduce some axioms that constrain the values of those functions: size never returns zero, bill never returns a map with a zero value and no product is a sub-product of itself. The latter involves an auxiliary function issub to determine if one product is a sub-product (immediate or not) of another. Below, dom applied to a map returns the set of all its arguments, $x$ is a Cartesian product and Bool is the type of the Boolean values with the usual logical operations. 
axiom

$(\forall$ p,q: Product • $\operatorname{size}(p)>0 \wedge \neg$ issub(p,p) $\wedge$ $q \in \operatorname{dom} \operatorname{bill}(p) \Rightarrow \operatorname{bill}(p)(q)>0)$

\section{value}

issub: Product $\times$ Product $\rightarrow$ Bool $\operatorname{issub}(q, p) \equiv q \in \operatorname{dom} \operatorname{bill}(p) \vee$

$(\exists$ r:Product $\bullet$ issub $(q, r) \wedge$ issub $(r, p))$

Consider an example product, a snow shovel (Vollmann, 1992) (Janowski, Moreira and Sousa, 2000). The picture below shows the product design on the left and the sub-product graph on the right, which includes the values of functions size (numbers in boxes) and bill (arrows and labels).
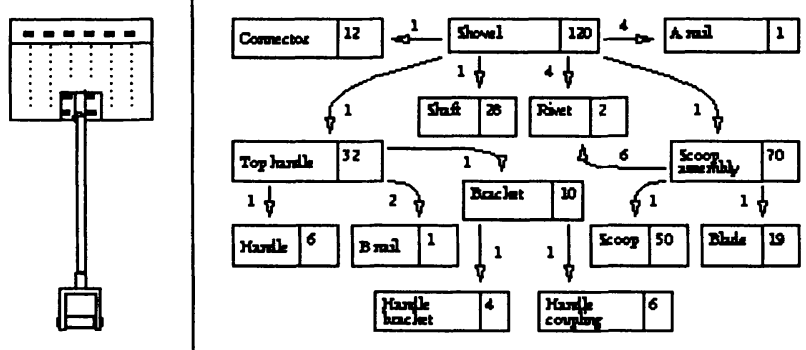

Production is carried out within a cell subject to the constraints on: the maximum number of products the cell can store in the warehouse (weighted by their storage requirements), the numbers of products in the warehouse (stocks), the set of products we can manufacture from their sub-products and the number of items manufacturable within a shift (shopfloor). Formally, we introduce an abstract type Cell and three corresponding functions space, stock and shop, such that the current occupancy of the warehouse is not greater than its capacity, a product that can be manufactured is non-atomic and the manufacturable quantity is at least one.

type
Cell

value

space: Cell $\rightarrow$ Nat,

stock: Cell $\times$ Product $\rightarrow$ Nat,

shop: Cell $\rightarrow$ (Product $\stackrel{m}{\longrightarrow}$ Nat) axiom

$(\forall \mathrm{c}:$ Cell $\bullet$

occupancy $(c) \leq \operatorname{space}(c) \wedge$

$(\forall \mathrm{p}:$ Product $\bullet \mathrm{p} \in \operatorname{dom} \operatorname{shop}(\mathrm{c}) \Rightarrow$ $\operatorname{shop}(c)(p) \neq 0 \wedge$ bill $(p) \neq[])$

Function occupancy adds all stocks in a cell, weighted by their storage requirements. We assume that space and shop are static attributes which only constrain production. There are three operations to change stocks: store increments the stock of a given product, deliver decrements the stock and manufacture increments the stock for the product and decrements the stocks for all its sub-products. They have the same type:

value

$$
\text { store, deliver, manufacture: Product } \times \text { Nat } \times \text { Cell } \stackrel{\sim}{\longrightarrow} \text { Cell }
$$

For instance, manufacture is defined using a pair of logical conditions: a precondition defines the range of argument values, a post-condition defines the relation between the arguments and the results, provided they satisfy a pre-condition. 
manufacture(p,n,c) as $\mathrm{c}^{\prime}$

post stock $\left(c^{\prime}, p\right)=\operatorname{stock}(\mathrm{c}, \mathrm{p})+\mathrm{n} \wedge \ldots$

$\left(\forall \mathrm{q}: \operatorname{Product} \bullet \mathrm{q} \in \operatorname{dom} \operatorname{bill}(\mathrm{p}) \Rightarrow \operatorname{stock}\left(\mathrm{c}^{\prime}, \mathrm{q}\right)=\operatorname{stock}(\mathrm{c}, \mathrm{q})-\mathrm{n}^{*} \operatorname{bill}(\mathrm{p})(\mathrm{q})\right)$

pre $p \in \operatorname{dom} \operatorname{shop}(c) \wedge n \leq \operatorname{shop}(c)(p) \wedge \ldots$

The type Operation includes all operations together with their arguments. Two functions are defined on this type: enough determines if a given cell has enough resources to execute an operation (the corresponding pre-condition holds) and exec executes the operation on the cell and returns a modified cell.

type

Operation $==$ store(Product, Nat)

deliver(Product, Nat) $\mid$ manufacture(Product,Nat)

value

enough: Operation $\times$ Cell $\rightarrow$ Bool

enough $(o p, c) \equiv$

if op=deliver(p,n)

then stock $(c, p) \geq n$ else ... end, exec: Operation $\times$ Cell $\stackrel{\sim}{\longrightarrow}$ Cell

$\operatorname{exec}(\mathrm{op}, \mathrm{c}) \equiv$ if $\mathrm{op}=\mathrm{store}(\mathrm{p}, \mathrm{n})$

then store $(p, n, c)$ else $\ldots$ end

pre enough(op,c)

The actual production is carried out by a sequence of such operations. We introduce the type Process and the functions enough and exec, to check if a cell has enough resources for a process and to execute a process, respectively. $h \boldsymbol{d}$ returns the first element of a non-empty list and $t l$ returns the list with the first element removed.

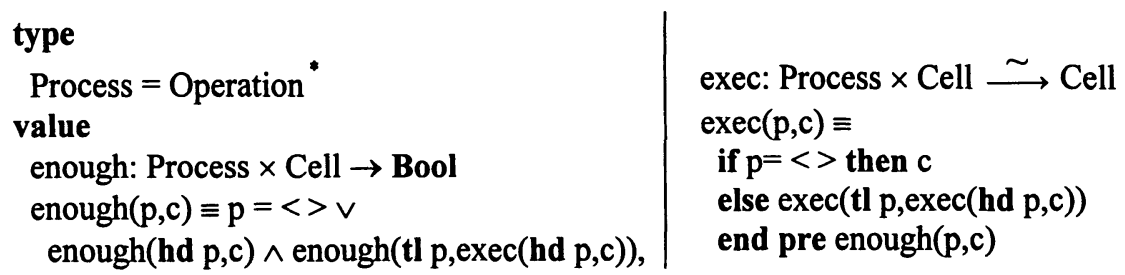

Consider a cell $c$ to produce snow shovels (Janowski, Moreira and Sousa, 2000). The following tables describe the values of the functions space, stock and shop when applied to this cell. In particular, the cell has the storage capacity of 200000, contains the stock of 150 shovels and can manufacture 100 items during a shift.

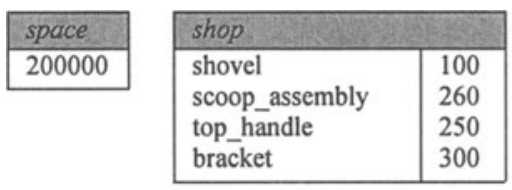

\begin{tabular}{|l|l|}
\hline stock \\
\hline shovel & 150 \\
scoop_assembly & 300 \\
rivet & 1600 \\
connector & 550 \\
top_handle & 400 \\
shaft & 150 \\
a_nail & 1200 \\
blade & 300 \\
scoop & 250 \\
\hline
\end{tabular}




\section{PLANNING DISTRIBUTED PRODUCTION}

Equipped with the notation and the basic concepts for modeling production, we can approach the main problem of this paper: planning distributed production. In the following sections we formulate the problem, propose a solution, analyze it informally and discuss a possible implementation.

\subsection{Problem Formulation}

The problem is to implement a given production goal using the resources offered by a set of cells. Each goal describes the product and the number of items to deliver, formally a value of the record type Goal with two corresponding fields prod and vol and the function revol which modifies a goal by changing its volume field.

type

Goal:: prod: Product vol: Nat $\leftrightarrow$ revol

Consider first a restricted case of the problem, for a single cell $c$. Given a goal $g$, the problem is to find a process $p$ such that $c$ has enough resources to execute $p$ and the execution results in the stock for $\operatorname{prod}(g)$ no less than vol$(g)$. The function satisfies formalizes this property while generate (defined implicitly) produces the required process, if one exists. Together, they formulate the problem for the single-cell case.

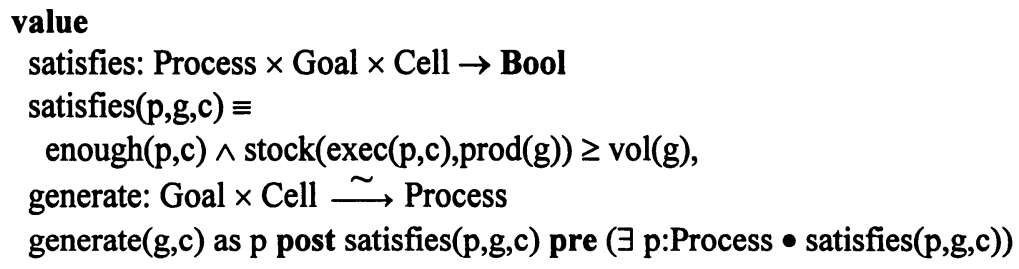

Consider a goal $g$, a set $c s$ of production cells and a distinguished cell $c \in c s$. We seek a process $p$ for $c$ that implements $g$. For all store operations in $p$, such an operation defines a sub-goal $g^{\prime}$, we also look for their implementation in $c s$, a cell $c^{\prime} \in c s$ and a process $p^{\prime}$ which implements $g^{\prime}$ when executed on $c^{\prime}$. Like $p, p^{\prime}$ may also contain sub-goals (sub-sub-goals with respect to $p$ ). To represent such executions involving multiple cells we introduce a "protocol", a process which links all store operations with their possible implementations (a cell and a protocol, recursively). We say the protocol is well formed with respect to the cell-set cs (function iswf) if every cell occurs in this protocol (and recursively any sub-protocol) at most once and is in cs.

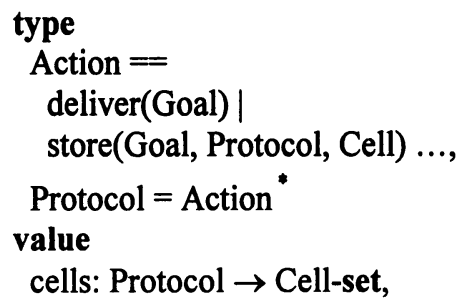

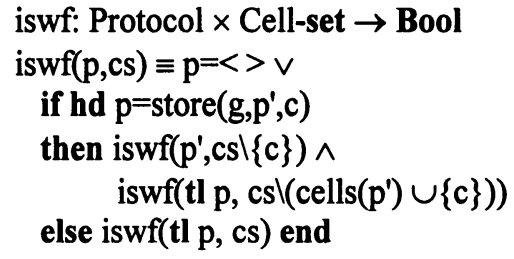


Then in order for the protocol $p$ and the cell $c \in c s$ to satisfy a goal $g$ the following conditions must be satisfied: (1) $c$ has enough resources to execute $p$, (2) the execution results in the stock for $\operatorname{prod}(g)$ greater than or equal to $\operatorname{vol}(g)$ and (3) every sub-goal $g^{\prime}$ in $p$ is satisfied by the corresponding cell $c^{\prime} \in c s$ and the protocol $p^{\prime}$. The function satisfies is defined recursively, as below. Function generate produces the required protocol, if one exists, defined using pre- and post-conditions.

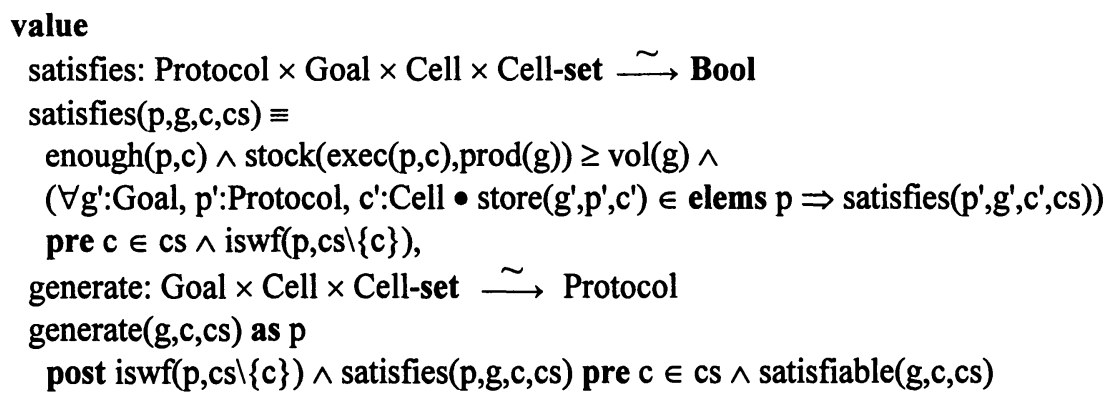

\subsection{Problem Solution}

We provide an explicit definition for the function generate, showing how it can be actually calculated. Like before, we start with the single cell case.

Given a goal $g$ and a cell $c$ the function first checks if the stock in $c$ already satisfies $g$. If so then we produce the empty process, otherwise we recalculate the volume by subtracting the existing stock. For the resulting goal $g$ ', if the product is not manufacturable then we generate the process with an operation to store the missing quantity. Otherwise we first generate a process $p 1$ to obtain the needed subproducts (gen_sub) then $p 2$ to manufacture the final product (gen fin) and finally concatenate such processes $\left(p 1^{\wedge} p 2\right)$. Function gen_fin takes into account the cell $c$ after it executed $p 1(\operatorname{exec}(c, p 1))$. Here is the explicit definition of generate:

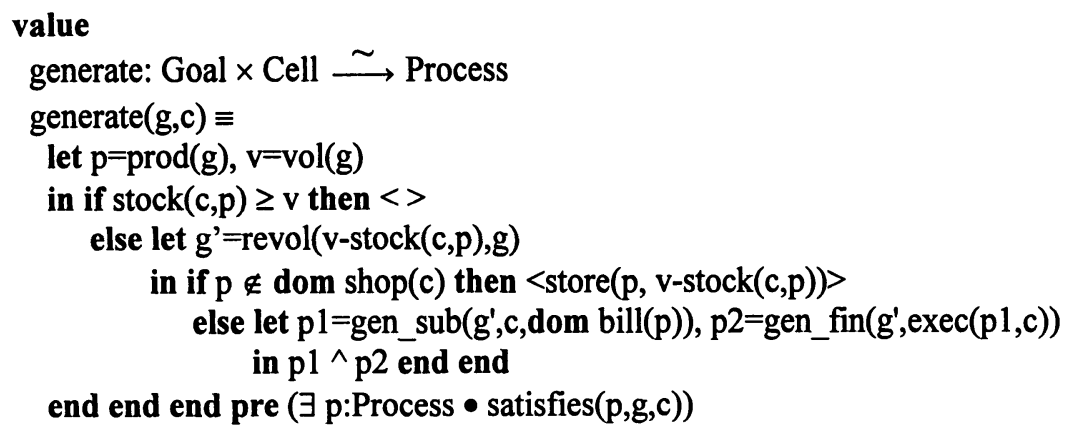

Function gen_fin generates a sequence of manufacture operations for $\operatorname{prod}(g)$ and either the maximum quantity allowed by the shopfloor $(\operatorname{shop}(c)(\operatorname{prod}(g)))$ or $\operatorname{vol}(g)$ (if less than this quantity), until the whole required volume is manufactured. Function gen sub considers each immediate sub-product of $\operatorname{prod}(g)$, calculates a 
(sub-) goal for this product according to the needs of $g$, recursively calls generate to produce a process for this goal, and concatenates all such processes.

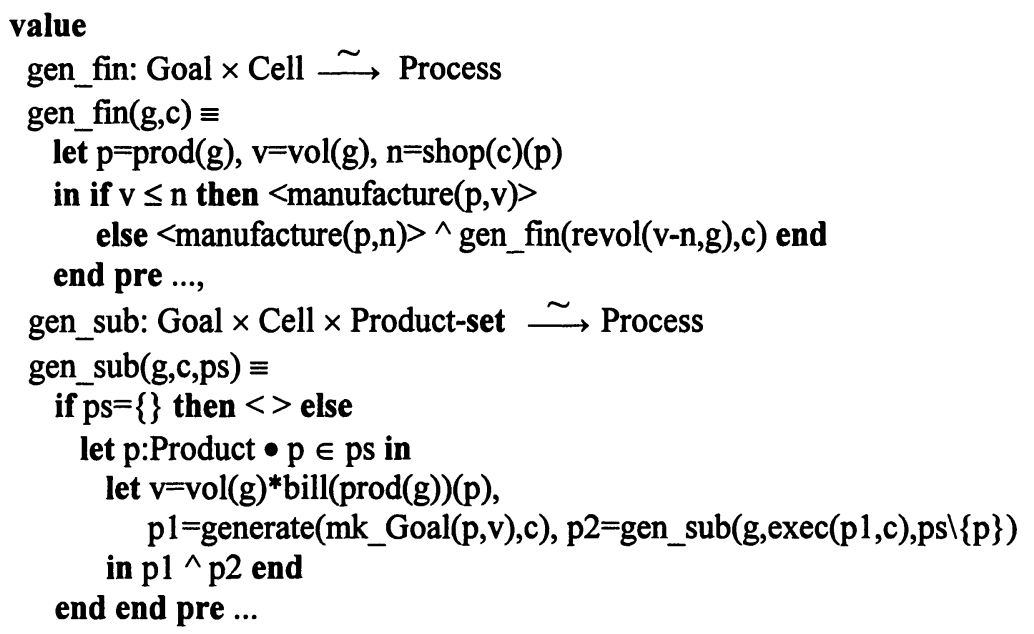

Consider the cell $c$ to produce snow shovels, as in Section 2. Below, we show some results produced by the generate function, applied to deliver various quantities of the shovel product. If the quantity is 150 then generate returns an empty process, the amount is already on stock. If the quantity is 250 then we still lack 100 items, which can be manufactured within one shift from the existing subproducts. If the quantity is 300 then we already need two shifts, one to manufacture 100 items and another 50 items; there is still enough sub-products. Raising the quantity to 450 , however, we miss 150 shafts which cannot be manufactured internally but must be obtained from outside. Finally, with the quantity raised to 500 , we miss 250 shafts, 200 a-nails and 50 scoop-assemblies. The latter can be manufactured but the added demand on rivets (for producing scoop-assemblies as well as shovels) exceeds the stock by 100 .
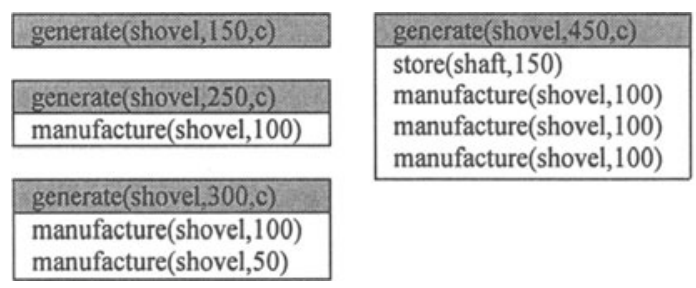

In the general case, consider a set $c s$ of cells, $c \in c s$ and a goal $g$. The main difference from the single-cell case is how we deal with the lack of resources in $c$ to satisfy $g$; when $c$ has insufficient stock to satisfy the volume of $g$ and it lacks the capacity to manufacture the missing number of $\operatorname{prod}(g)$. First we assume (by a precondition on generate) that there exists at least one cell in $c s$ for which $g$ is satisfiable. Second, we take any cell $c^{\prime} \in c s$ with this property, recursively generate a protocol $p$ for $c^{\prime}$ and produce a corresponding store action where $g, p$ and $c^{\prime}$ occur explicitly. Otherwise, the generate function is similar as before: 


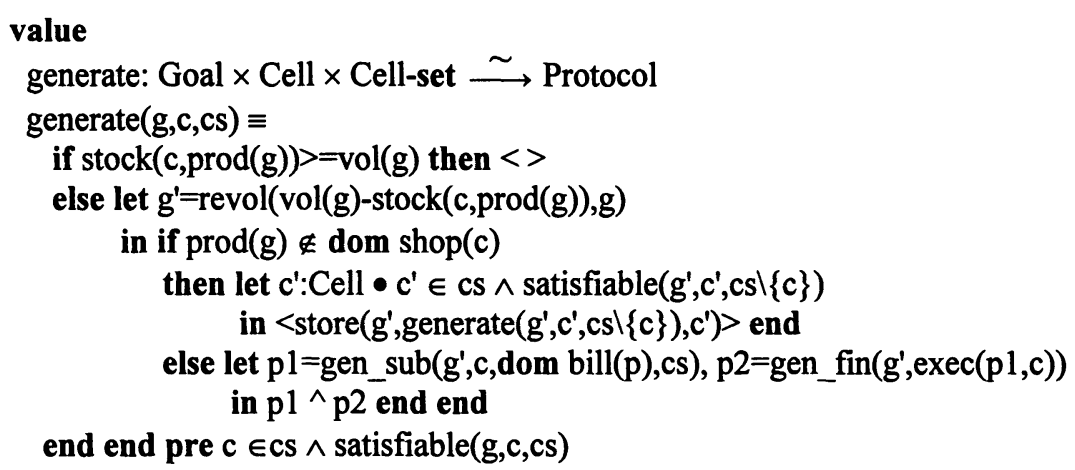

gen_fin is as before, it generates the manufacture operations for $c$ after it has executed $p l(\operatorname{exec}(p l, c))$ but ignoring the effect of $p l$ on other cells. gen_sub is also similar, except it now returns a protocol (rather than a process) and takes the set $c s$ of cells as one more argument; in order to pass it to the function generate. It also follows on the state-changes in $c$ but not how other cells change: as soon as the cell is used in the protocol for a sub-product it is not considered for the remaining subproducts (the iswf condition for the well-defined protocol).

\subsection{Protocol Correctness}

We argue about the correctness of the solution: that the model is well defined, that it is internally consistent, and that it provides the correct solution to our problem.

The first part we can check fully automatically. The RSL type checker will parse all specifications, decide if they are syntactically correct and obey the typing constraints. For instance, it will detect that the precondition for the manufacture function applies stock in the wrong order of its arguments and the generate function tries to concatenate a protocol with a process. The latter requires explicit conversions from Protocol to Process (flat) and vice versa (nest), applied whenever we want to execute a protocol on a given cell $(\operatorname{exec}(f l a t(p), c))$ or concatenate the two (p1^nest(p2)). Except for those two errors, the models are checked successfully.

The second part involves checking in particular that all functions are applied within their preconditions. This task cannot be fully automated as it would involve reasoning about the truth of arbitrary logical expressions. Instead, the type checker generates some confidence conditions to inspect. There are 47 conditions generated for the specifications in this paper, most of which are discarded immediately. For instance: $\operatorname{bill}(p)(q)>0$ requires checking that $q$ is in the domain of $\operatorname{bill}(p)$, decreasing the stock in the manufacture function requires checking that the result is positive, using the let expression to choose a cell satisfying the goal (in the generate function) requires checking that such a cell exists. And so on.

The third part is to argue that the proposed solution correctly solves the problem, that the explicit definition of generate satisfies the implicit definition; the postcondition produces a logically true statement: satisfies(generate(g,c,cs),g,c,cs) for any goal $g$, set of cells $c s$ and $c \in c s$, such that $g$ is satisfiable by $c$ and $c s$. Given that satisfies is defined recursively, we justify this by induction: if the statement is true 
for all operations store $\left(g^{\prime}, p^{\prime}, c^{\prime}\right)$ in the generated protocol then it is true for the protocol itself. We demonstrate that: (1) $c$ has enough resources to execute the generated protocol and (2) the resulting stock for $\operatorname{prod}(g)$ satisfies the required volume $\operatorname{vol}(g)$. Clearly, the protocol is generated to satisfy both conditions: we first prepare the required quantities of all sub-products of prod(g) (gen_sub) then generate the sequence of manufacture operations (making sure the volumes do not exceed the shopfloor capacity) to produce $\operatorname{vol}(g)$ items of $\operatorname{prod}(g)$. If the required product is not manufacturable and the stock does not satisfy the required volume then we delegate the goal (minus the existing stock) to some other cell. We can see that (1) is satisfied as we make sure the resources exist before we try to use them as well as (2) holds with the stock value exactly equal to the required volume.

\subsection{Email-Based Implementation}

The implementation is based on the environment for simulating production systems. Each cell has a concrete representation using text files: the STOCKS file describes product stocks, the STATIC file describes the warehouse/shopfloor constraints and the PRODUCTS file contains product information. Given such files, we implement the operations on the cell (store, deliver and manufacture) as text transformations, which read all three files above and modify the STOCKS file; we implemented them with the Awk language (Robbins, 1989). Similar text files are used to document a process, so the exec and enough functions can be implemented as simple shell scripts. In particular exec looks through the process file and for each entry calls the corresponding Awk program. We assume that each cell has an email address to communicate with other cells, with two additional files to facilitate communication: aliases defines the mail alias cells with the addresses of all active cells and forward causes each received email is to be sent to the script incoming for further processing.

The implementation for the protocol is the program generate, available to run for each cell. Initially, the program is called from the command line, given a goal as input. Then it carries out processing of this goal, taking into account the local STOCKS and STATIC files and printing the generated operations on the screen. The processing is like described by the function generate. There are two obstacles, however, both related to the use of an implicit let expression (take an arbitrary product/cell satisfying a certain property). In gen_sub, the implementation looks through the PRODUCTS file to produce the first product satisfying the property.

In generate, the let expression is used to choose a cell which can satisfy a given goal. The implementation broadcasts the goal to all active cells. When a cell receives such a message it calls the local generate program, then sends back the result of this invocation. The replies are gathered in a file: either a process description that tells how to implement the goal or an indication that the goal is not satisfiable. When all replies have been received and all failed to produce a result then generate also outputs the failed message and quits. Otherwise it works as follows: (1) accept one of the received solutions, (2) broadcast a message to cells informing them about the choice, (3) calculate all cells in the solution and remove them from the cells alias, (4) print the choice on the screen and (5) continue as in generate. There are many ways to reach a decision (1): the first solution received, the "best" solution received, the solution coming from the top-priority cell (according to some ranking). The details of the implementation will be subject of a companion paper. 


\section{CONCLUSIONS}

We focused in this paper on the specific problem related to the design of virtual organizations: how to distribute production among a set of cells to satisfy a given production goal. We formalized this problem, proposed a solution, argued about correctness and outlined an implementation. The model for production follows (Janowski, Lugo and Zheng, 1999) and the simulation environment have been described in (Janowski, Moreira and Sousa, 2000).

The concept of a virtual organization has received a lot of attention. We have seen reports on case studies (Molina and Flores, 1999), architectures (CamarinhaMatos and others, 1997), standards (Clements, 1997), protocols (Hardwick and others, 1997) and supporting technologies (Camarinha-Matos and Afsarmanesh, 1997). Also planning and design issues has been approached (Schonsleben and Buchel, 1998), albeit in an informal way. This paper is different in the sense of adopting a formal approach to modeling, used to capture a specific design-related problem, then propose and justify a solution to this problem.

We have several plans to continue this work. We want to document in more detail the implementation of the protocol, including its applications to some concrete problems. To gain the confidence in our design we intend to prove that this protocol is correct, with formal rather than informal arguments. We want to extend the problem with timing attached to production goals: the maximum number of shifts from placing the order to its final delivery. This, in turn, opens the possibility for accepting some imperfect (perhaps violating the deadline) but still satisfactory (producing the required volume of the product) solutions.

\section{REFERENCES}

1. Camarinha-Matos LM and Afsarmanesh H. Handbook of Life Cycle Engineering, Virtual Enterprise: Life Cycle Supporting Tools and Technologies. Chapman and Hall, 1997.

2. Camarinha-Matos LM, Afsarmanesh H, Garita C and Lima C. Towards an Architecture for Virtual Enterprise. World Congress on Intelligent Manufacturing, 1997.

3. Clements P. Standards Support for the Integration and Interoperation of the Virtual Enterprise. Enterprise Integration Modeling Technology, 1997.

4. Handfield R and Nichols E. Supply Chain Management. Prentice Hall, 1999.

5. Hardwick M, Rando T, Spooner DL and Morris KC. Data Protocols for the Industrial Virtual Enterprise. IEEE Journal of Internet Computing, 1997, vol. 1, no. 1.

6. Janowski T, Lugo $\mathrm{G}$ and Zheng $\mathrm{H}$. Modeling an Extended/Virtual Enterprise by the Composition of Enterprise Models. Journal of Intelligent and Robotic Systems, 1999, vol. 26, no. 2-3, 303-324.

7. Janowski T, Moreira F and Sousa R. Production Modeling as Shell Programming: Concurrency and Delegation. IFAC Symposium on Manufacturing, Modeling, Management and Control, Rio, Greece, July 2000. Elsevier.

8. Molina A and Flores M. A Virtual Enterprise in Mexico: From Concepts to Practice. Journal of Intelligent and Robotic Systems, 1999, vol. 26, no. 2-3, 289-302.

9. Robbins AD. Effective Awk Programming. Free Software Foundation, 1989.

10. Schonsleben $P$ and Buchel A. Organizing the Extended Enterprise. Chapman and Hall, 1998.

11. Vernadat F. Enterprise Modeling and Integration. Chapman and Hall, 1996.

12. Vollmann TE. Manufacturing, Planning and Control Systems. Irwin, 1992.

13. The RAISE Method Group. The RAISE Specification Language. Prentice Hall, 1992. 